\section{The key points for treatment of Parkinsonism in older persons}

\author{
Fulvio Lauretani, ${ }^{1,2}$ Andrea Ticinesi, 1,2 \\ Tiziana Meschi, 1,2 Marcello Maggio 2,3 \\ ${ }^{1}$ Internal Medicine and Critical Subacute \\ Care Unit, Laboratory of Disability \\ Prevention, Department of Geriatrics and \\ Rehabilitation, University-Hospital of \\ Parma; ${ }^{2}$ Department of Clinical and \\ Experimental Medicine, University of \\ Parma; ${ }^{3}$ Department of Clinical Geriatrics \\ and Geriatric Rehabilitation, University- \\ Hospital of Parma, Italy
}

\section{Abstract}

Signs and symptoms of Parkinsonism have a high prevalence in older persons. Parkinsonism is associated with negative outcomes in the elderly and there is still uncertainty about when and how to start levodopa in these conditions. The diagnosis of idiopathic Parkinson disease is often not pursued in the oldest old.

The coexistence of both motor and cognitive impairment is the strongest factor limiting the initiation of treatment with levodopa and/or dopamine agonists in a geriatric setting, given the possibility of producing psychotic symptoms, such as visual hallucinations.

It seems reasonable to perform at least one attempt to administer levodopa in older persons with parkinsonism, especially when symptoms and motility disorders are evident, in order to try to obtain an improvement in walking speed and balance. Important signs that should guide treatment for Parkinsonism in older persons are the presence of line-pipe rigidity and cogwheel rigidity.

\section{Short communication}

Signs and symptoms of Parkinsonism have a high prevalence in older persons, although often neglected. Parkinsonism is associated with negative outcomes in the elderly and there is still uncertainty about when and how to start levodopa in these conditions. ${ }^{1}$ The diagnosis of idiopathic Parkinson disease (PD) is often not pursued in the oldest old.

The coexistence of both motor and cognitive impairment is the strongest factor limiting the initiation of treatment with levodopa and/or dopamine agonists in a geriatric setting, given the possibility of producing psychotic symptoms, such as visual hallucinations. ${ }^{1}$

However, it seems reasonable to perform at least one attempt to administer levodopa in older persons with parkinsonism, especially when symptoms and mobility disability are evident, in order to try to obtain in them an improvement in walking speed and balance, with a possibility to reduce risk of falling in this category of patients prone to fragility fracture.

Actually, extra-pyramidal signs could potentially be separated to the age-related motoric signs, using standardized tests and cut-offs values for evaluating, for example, bradikinesia (slowness of movement) ${ }^{2}$ and decreased movement amplitude. ${ }^{3}$

From our point of view new criteria for identifying Parkinson's disease even in older persons are clinically relevant for reducing diagnostic errors in the diagnosis of PD in the elderly, emphasizing the importance of extrapyramidal signs, such as bradikinesia and the presence of line-pipe rigidity and cogwheel rigidity, ${ }^{3}$ that should guide treatment for parkinsonism in older persons.

The definition of these symptoms has been recently highlighted in the consensus realized by the Movement Disorder Society (MDS) Task Force. ${ }^{2}$ Briefly, bradykinesia is defined as combination of slowness and decreased movement amplitude while the rigidity or lead-pipe rigidity is defined as: the presence of slow passive movement of major joints with the patient in a relaxed position and the examiner manipulating the limbs and neck. This movement should be velocity-independent, with the resistance to passive movement not reflecting failure to relax. The authors also suggested that isolated cogwheeling without lead-pipe rigidity does not fulfill minimum requirements for rigidity in PD. Typical rest tremor, which is suppressed during movement initiation, is also included in the diagnosis of parkinsonism with bradikinesia and lead-pipe rigidity, while postural instability is important but not necessary sign for the diagnosis of PD. ${ }^{2}$

In the new diagnostic criteria for PD a different degree of extra-pyramidal signs is emphasized, with bradykinesia, defined as slowness movement but also decreased amplitude of movement, as the most important feature, obtaining the dignity of a sine qua non sign. At least two out of four cardinal symptoms should be present for establishing the diagnosis, ${ }^{2}$ and in particular bradikinesia plus rigidity or resting tremor thus, rigidity, which is extremely common among older persons, has gained the same dignity of the other motor symptoms typical of PD (Figure 1).

These findings suggest that these signs could guide the decisions of prescribing levodopa and establishing its daily dosages in geriatric clinical practice. After the identification of established Parkinsonism, the MDS clinical diagnostic criteria for Parkinson's disease also emphasized absolute exclusion cri-
Correspondence: Fulvio Lauretani, Internal Medicine and Critical Subacute Care Unit, Laboratory of Disability Prevention, Department of Geriatrics and Rehabilitation, University Hospital of Parma, via Gramsci 14, 43100 Parma, Italy.

Tel.: +39.0521.704869 - Fax: +39.0521.704869.

E-mail: flauretani@ao.pr.it

Key words: Parkinsonism; cognitive impairment; cogwheel rigidity; lead-pipe rigidity.

Contributions: the authors contributed equally.

Conflict of interest: the authors declare no potential conflict of interest.

Received for publication: 12 July 2016.

Revision received: 4 November 2016.

Accepted for publication: 25 November 2016.

This work is licensed under a Creative Commons Attribution-NonCommercial 4.0 International License (CC BY-NC 4.0).

(C) Copyright F. Lauretani et al., 2016

Licensee PAGEPress, Italy

Geriatric Care 2016; 2:6156

doi:10.4081/gc.2016.6156

teria to establish clinically PD, such as observing a normal functional neuroimaging of the presynaptic dopaminergic system. In particular, if we have a positive DaTSCAN SPECT (single-photon emissi on computed tomography) the diagnosis of PD or other atypical Parkinsonism is always possible, while if we observe a normal DaTSCAN SPECT the diagnosis of PD is not probable. A recent study by our research group, including data from a large number of brain DaTSCAN SPECTs, that allow a functional evaluation of the presynaptic dopaminergic system, showed that the presence of plastic rigidity and bradikinesia are the most important signs associated with a positive imaging.${ }^{4}$ However, more studies are necessary to clearly establish the relationship between the presynaptic dopaminergic deficiency evaluated on dopamine transporter imaging and the utilization of subsequent medication, even if preliminary results seem to confirm a good correlation between putamen DAT binding and bradykinesia, while are not associated putamen DAT binding and different degree of plastic rigidity. ${ }^{5}$

Abnormal neurological signs without clear evidence of major neurological diseases are often present in older individuals. A possible definition for each neurological sign in the light of the age-associated changes of the nervous system has been previously provided. ${ }^{5}$ Therefore, the importance of neurological examination in case of suspected PD should be emphasized. ${ }^{6}$

At the moment several population based 
studies are evaluating the factors differentiating Parkinson's disease from normal aging, ${ }^{7,8}$ but it is still not clear which symptoms might be really specific of Parkinson's disease or mask minor changes with aging.

Our clinical experience with geriatric patients confirms the importance of bradikinesia and rigidity as key signs that may guide the initiation of levodopa or dopamine agonists, especially when no overt cognitive impairment is present. On the other side, bradykinesia alone, the pivotal sign of extra-pyramidal disease, is not sufficiently accurate for diagnosing PD in older persons. In fact, it can be easily confused with reduction of walking speed, a very common condition in the elderly. ${ }^{9}$ Given the epidemiology of PD, with incidence and prevalence maximal in older persons, we suggest that in the diagnostic and therapeutic management of this disease both the neurologist and the geriatrician could be involved. When the lead-pipe or the cogwheel are present, the basal ganglia are instead inevitably compromised and a diagnosis of Parkinsonism is very probable. ${ }^{10}$ Thus, starting a trial with levodopa could be recommendable in this category of patients, since even minimal improvements in walking speed and rigidity may result in significant advantages in terms of ability to perform the activities of daily living.

The only contraindication to initiate levodopa in this clinical setting could be the presence of moderate-severe cognitive impairment or history of psychosis and high risk of delirium, and also attention for the presence of hypotension that could be an important side effect limiting the use of levodopa in parkinsonism patients ${ }^{11}$ especially if the physician suspect an atypical parkinsonism with less levodopa responsivity.

L-dopa is usually contraindicated in presence of dementia because the risk of agitation or hallucination (for example in progressive supranuclear palsy patients or Alzheimer patients with mild parkinsonism). However some dementia with Lewy bodies patients might benefit of L-dopa in terms of motor and cognitive dysfunction and a trial with low-dose of L-dopa could be considered in these patients. ${ }^{12,13}$

A comprehensive geriatric assessment should therefore be performed, in order to exclude moderate or severe dementia and to accurately identify possible risks and benefits of dopaminergic treatment.

Given the epidemiology of this disease, with incidence and prevalence maximal in older persons, we suggest that in the diagnostic and therapeutic management of this disease both the neurologist and the geriatrician could be involved. ${ }^{14}$ The clinical approaches suggested by the MDS Task Force could open new ways of managing elderly patients with slow walking speed and muscular rigidity and established diagnosis of Parkinsonism. In fact physicians could really prevent mobility disability in older persons, where pathologies of the nervous system are often under-recognized and not unavoidable consequence of the aging process.

The multidisciplinary integration of comprehensive geriatric assessment with neurologist could be successful for the management of older persons with both overt PD and isolated extra-pyramidal symptoms.

\section{References}

1. Lauretani F, Ceda GP, Pelliccioni P, et al. Approaching neurological diseases to reduce mobility limitations in older persons. Curr Pharm Des 2010;20:149-64.

2. Postuma RB, Berg D, Stern M, et al. MDS clinical diagnostic criteria for Parkinson's disease. Mov Disord 2015;30:1591-601.

3. Ghiglione P. Cogwheel rigidity. Arch Neurol 2005;62:829-30.

4. Lauretani F, Ruffini L, Scaglioni A, et al. Utilization of the DaT-SCAN SPECT in the diagnosis of Parkinson's disease in older subjects. Lett Drug Design Discov 2015;8: 614-21.

5. Nissen T, Malek N, Grosset KA, et al. Baseline [(123) I]FP-CIT SPECT (DaTSCAN) severity correlates with medication use at 3 years in Parkinson's disease. Acta Neurol Scand 2014;129:204-8.

6. Bloem BR, Brundin P. How I examine my patient: the art of neurological examination for Parkinson's disease. J Parkinsons Dis 2014;4:563-5.

7. Mahlknecht P, Kiechl S, Stockner H, et al. Predictors for mild parkinsonian signs: a prospective population-based study. Parkinsonism Relat Disord 2015;21:321-4.

8. Lerche S, Hobert M, Brockmann K, et al. Mild parkinsonian signs in the elderly--is there an association with PD? Crossectional findings in 992 individuals. PLoS One 2014;27:e92878.

9. Ferrucci L, Bandinelli S, Cavazzini C, et al. Neurological examination findings to predict limitations in mobility and falls in older persons without a history of neurological disease. Am J Med 2004;116:807-15.

10. Berardelli A, Sabra AF, Hallett M. Physiological mechanisms of rigidity in Parkinson's disease. J Neurol Neurosurg Psychiatry 1983;46:45-53.

11. Noack C, Schroeder C, Heusser K, Lipp A. Cardiovascular effects of levodopa in Parkinson's disease. Parkinsonism Relat Disord 2014;20.815-8.

12. Lucetti C, Logi C, Del Dotto P, et al. Levodopa response in dementia with lewy bodies: a 1-year follow-up study. Parkinsonism Relat Disord 2010;16:522-6.

13. Costa A, Peppe A, Mazzù I, et al. Dopamine treatment and cognitive functioning in individuals with Parkinson's disease: the "cognitive flexibility" hypothesis seems to work. Behav Neurol 2014;14:260896.

14. Lauretani F, Ticinesi A, Meschi T, et al. Randomized controlled trials for Alzheimer disease and Parkinson disease. Front Biosci 2016;8:378-89.
Figure 1. Rigidity is one of the typical symptoms of Parkinson disease (PD). MDS, Movement Disorder Society. 